\title{
Comparative Analysis of the Difference of the Vegetation Indexes between FY-3A/VIRR, FY-3A/MERSI and Terra/MODIS data
}

\author{
M.X. Ge \\ College of Computer Science and Technology, Chongqing University of Posts and Telecommunications, \\ Chongqing, China
}

Chinese Academy of Sciences Institute of Remote Sensing and Digital Earth State Key Laboratory of Remote Sensing, Beijing, China

J. Zhao

College of Computer Science and Technology, Chongqing University of Posts and Telecommunications, Chongqing, China

B. Zhong \& A.X. Yang

Chinese Academy of Sciences Institute of Remote Sensing and Digital Earth State Key Laboratory of Remote Sensing, Beijing, China

ABSTRACT: In order to demonstrate the feasibility of cooperative inversion of the vegetation indexes between FY-3A/VIRR, FY-3A/MERSI and Terra/MODIS, the NDVI from these data was compared. The authors selected 2012 year's data of FY-3A/VIRR, FY-3A/MERSI and Terra/MODIS which is covering the upstream of the Heihe and less cloudiness. The statistical method was used to study correlation of NDVI from the selected data. The result shows that there are significant correlations of NDVI from the data of FY3A/VIRR, FY-3A/MERSI and Terra/MODIS, and all the correlation coefficients exceeded 0.99.But significant differences are also exist between these data. The causes of NDVI differences were analyzed in detail, and found that spectral response function and radiometric calibration have a certain impact on vegetation index. These analyzes can provide a reference for cooperative inversion of multi-source remote sensing data.

KEYWORD: FY-3A/VIRR; FY-3A/MERSI; Terra/MODIS; NDVI; Comparative analysis; Cooperative inversion; The cause of difference

\section{INTRODUCTION}

FY-3A was launched from Taiyuan in Shanxi Province on 27 May 2008, which is the second generation polar-orbiting meteorological satellite series of China. It is to provide three-dimensional, quantitative, multi-spectrum global remote sensing data under global, all weather conditions, and all time conditions of continuous observation (Gallok et al, 2005). There are 11 observation instruments aboard this satellite. The visible and infrared radiometer (VIRR) is inherited observation instrument, and the medium resolution spectral imager (MERSI) is the first time aboard the satellite (Jun, 2009). Terra was launched on 18 December 1999, which is in a series of satellites among earth observing system, and it is a morning satellite. The satellite has carried a moderate resolution imaging spectrometer (MODIS). MODIS used on-orbit calibration, and it has stable performance and good calibration. MODIS has used to long-term observe the surface, biosphere, the solid Earth, atmosphere and oceans. Vegetation plays an important role in the Earth's ecosystems, and it is an important indicator of the environmental impact produced by climatic and human factors. Vegetation index is an important parameter to describe the characteristics of vegetation (Kaufman Y J et al, 1992), and long time series of vegetation index plays an important role in the terrestrial ecosystem monitoring and modeling. Normalized difference vegetation index (NDVI) is one of the more commonly used vegetation index. In order to promote the construction of FY-3A application system, and provide a reference for the application of multi-source remote sensing data, the authors compared NDVI of FY3A/VIRR, FY-3A/MERSI and Terra/MODIS, and analyzed the causes of NDVI differences from these data in detail.

\section{METHODLOGY}

\subsection{Study area and data source}

The study area is near the upstream of the Heihe Watershed. Its latitude and longitude are E100 $15^{\prime}-$ $100^{\circ} 45^{\prime}, 38^{\circ} 45^{\prime}-39^{\circ} 5^{\prime}$. The land use type is mainly for arable land. The L1 data products of FY3A/VIRR and FY-3A/MERSI are provided by the National Satellite Meteorological Center site. The data of Terra/MODIS is Level 1B MOD021KM data products and its corresponding geographic coordinates MOD03 data products are produced by NASA Data Archive Center site. MOD021KM data products as for earth observation data is corrected $1 \mathrm{~km}$ 
resolution, including the re-sampled band (Tomoaki M. 2000), such as the $250 \mathrm{~m}$ and $500 \mathrm{~m}$ data. Those data products are less cloudiness data which covering the upstream of the Heihe Watershed transiting on the same day. The time span is from the January 2012 to December 2012, which can ensure the impact of the atmospheric conditions (Xueyi Z. 2009), solar elevation angle and solar azimuth angle are basically the same on the selected data. The spectral range of FY-3A/VIRR is $0.43 \sim 12.5 \mu \mathrm{m}$. It has 10 channels. The scan range is $\pm 55.4^{\circ}$. The ground resolution is $1.1 \mathrm{Km}$. The spectral range of FY3A/MERSI is $0.41 \sim 12.5 \mu \mathrm{m}$, which has 20 channels. The scanning range is $\pm 55.4^{\circ}$. The ground resolution is $250 \mathrm{~m}$ and $1000 \mathrm{~m}$. The spectral range of Terra/MODIS is $0.4 \sim 14 \mu \mathrm{m}$, which has 36 channels. The ground resolution is $250 \mathrm{~m}, 500 \mathrm{~m}$ and $1000 \mathrm{~m}$. The scanning width is $2330 \mathrm{~km}$. The selected band of Normalized Difference Vegetation Index is red band that has strong absorption of green plants and nearinfrared band that has high reflectivity and high transmission for green plants. For the above reasons, the first and second channels of Terra/MODIS, the first and second channels of FY-3A/VIRR and the third and fourth channels of FY-3A/MERSI has been selected. The red and near-infrared channel settings of these sensors are shown in Table 1.

Table 1. Comparison of correspond band between Terra/MODIS, FY-3A/VIRR and FY-3A/MERSI

\begin{tabular}{cccccc}
\hline \multicolumn{2}{c}{ Terra/MODIS } & \multicolumn{2}{c}{ FY-3A/VIRR } & \multicolumn{2}{c}{ FY-3B/MERSI } \\
\hline band & wavelength $(\mu \mathrm{m})$ & band & wavelength $(\mu \mathrm{m})$ & band & wavelength $(\mu \mathrm{m})$ \\
Band1 & $0.620-0.670$ & Band1 & $0.580-0.680$ & Band3 & $0.600-0.700$ \\
Band2 & $0.841-0.876$ & Band2 & $0.840-0.890$ & Band4 & $0.815-0.915$ \\
\hline
\end{tabular}

where $\rho_{\text {MODIS }}=$ the apparent reflectance of Terra/MODIS; $D N=$ earth observation count value; re-

\subsection{Data processing}

\subsubsection{Geometric correction}

Take Terra/MODIS data as basemap for geometric correction of FY-3A/VIRR and FY-3A/MERSI data, and the correction accuracy is controlled within one pixel.

\subsubsection{Crop, splice and projection conversion}

Since the repeat transition of Terra/MODIS is a period of 16 days. Every transition remote sensing data would not include all the study area. So the crop, splice and projection conversion of data which is cover the study area is needed. The original projection of MODIS is Sinusoidal Grid, it needs to be converted to UTM projection. The paper used MRT Swath which is provided by NASA website to process the data of Terra/MODIS, and used ENVI to realize the process of the data of FY-3A/VIRR and FY-3A/MERSI.

\subsubsection{Radiometric calibration}

The DN value of remote sensing data is earth observation count value, which needs to be radiometric calibration through calibration coefficients of the data. The change in solar zenith angle also makes some bias to the apparent reflectance, which specifically showing apparent reflectance has larger deviation along with the increase of solar zenith angle. So the apparent reflectance should be corrected according to solar zenith angle.

The apparent reflectance of Terra/MODIS is calculated as follows:

$\rho_{\text {MODIS }}=\frac{\text { reflectance_scales } *(D N-\text { reflectance_offsets })}{\cos \theta}$ flectance scales $=$ apparent reflectance gain coefficient; reflectance offsets = apparent reflectance offset coefficient; $\theta=$ Solar Zenith Angle.

The apparent reflectance of FY-3A/VIRR is calculated as follows:

$$
\begin{aligned}
& \rho_{\text {VIRR }}=\frac{S^{*} D N+I}{100 \cos \theta} * d^{2} \\
& d=1+0.0167 \sin \left(2 \pi \frac{\text { days }-93.5}{360}\right)
\end{aligned}
$$

where $\rho_{\text {VIRR }}=$ the apparent reflectance of FY3A/VIRR; $S=$ slope; $I=$ intercept; $d=$ the distance from the earth to the sun; days = acquisition date of the remote sensing data in the number days of the year.

The apparent reflectance of FY-3A/MERSI is calculated as follows:

$\rho_{\text {MERSI }}=\frac{k 0+k 1 * D N+k 2 * D N^{2}}{100 \cos \theta} * d^{2}$

where $\rho_{\text {MERSI }}=$ the apparent reflectance of FY$3 \mathrm{~A} / \mathrm{MERSI} ; k_{0}, k_{1}$ and $k_{2}=$ calibration coefficients.

\subsubsection{Calculation of NDVI}

The apparent reflectance of FY-3A/VIRR, FY3A/MERSI and Terra/MODIS should be calculated to normalized difference vegetation index.

The NDVI is calculated as follows:

$$
N D V I=\frac{\rho_{\text {nir }}-\rho_{\text {red }}}{\rho_{\text {nir }}+\rho_{\text {red }}}
$$

where $\rho_{\text {red }}=$ apparent reflectance of red band; $\rho_{\text {nir }}$ $=$ apparent reflectance of near-infrared band. 


\section{COMPARISON OF THE VEGETATION INDEXES}

Dynamic range and daily average of NDVI, FY3A/VIRR and FY-3A/MERSI deviation with Ter$\mathrm{ra} /$ MODIS's NDVI are as shown in table 2. Daily average of NDVI is as shown in figure 1.We can see that vegetation indexes of FY-3A/VIRR, FY3A/MERSI is very close to that of Terra/MODIS. They have a common trend and a significant correlation. All the correlation coefficients exceeded 0.99 . But there are some differences of NDVI between FY-3A/VIRR, FY-3A/MERSI and Terra/MODIS. Specific performance: First, dynamic range of FY3A/VIRR and FY-3A/MERSI is less than Ter$\mathrm{ra} / \mathrm{MODIS}$. The maximum value of FY-3A/VIRR and FY-3A/MERSI are 0.712 and 0.709 , however the maximum value of Terra/MODIS is up to 0.765 . Second, NDVI of Terra/MODIS is higher than that of FY-3A/VIRR and FY-3A/MERSI. Vegetation index of Terra/MODIS is more sensitive, and it can get more rich information of vegetation.

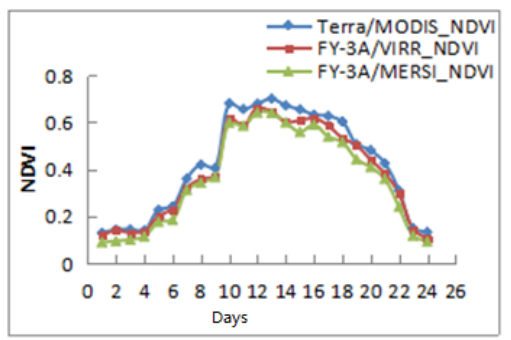

Figure 1. NDVI of Terra/MODSI, FY-3A/VIRRandFY3A/MERSI

Table 2. Comparison of NDVI between Terra/MODIS, FY-3A/VIRR and FY-3A/MERSI

\begin{tabular}{|c|c|c|c|c|c|c|c|c|}
\hline \multirow{2}{*}{$\begin{array}{l}\text { Data (year- } \\
\text { month-day) }\end{array}$} & \multicolumn{3}{|c|}{ dynamic range of NDVI } & \multicolumn{3}{|c|}{ daily average of NDVI } & \multicolumn{2}{|c|}{ deviation } \\
\hline & MODIS & VIRR & MERSI & MODIS & VIRR & MERSI & VIRR & MERSI \\
\hline 2012_01_10 & 0.154 & 0.130 & 0.113 & 0.130 & 0.120 & 0.090 & 0.010 & 0.040 \\
\hline $201201-11$ & 0.180 & 0.178 & 0.105 & 0.144 & 0.140 & 0.095 & 0.004 & 0.049 \\
\hline 2012_01_16 & 0.185 & 0.167 & 0.113 & 0.145 & 0.131 & 0.101 & 0.014 & 0.044 \\
\hline 2012_02_15 & 0.172 & 0.169 & 0.122 & 0.140 & 0.137 & 0.114 & 0.003 & 0.026 \\
\hline 2012_05_13 & 0.276 & 0.220 & 0.213 & 0.228 & 0.202 & 0.177 & 0.026 & 0.051 \\
\hline 2012_05_15 & 0.301 & 0.295 & 0.224 & 0.242 & 0.231 & 0.185 & 0.011 & 0.057 \\
\hline 2012_06_10 & 0.423 & 0.380 & 0.362 & 0.360 & 0.321 & 0.312 & 0.039 & 0.048 \\
\hline 2012_06_11 & 0.469 & 0.454 & 0.438 & 0.420 & 0.359 & 0.344 & 0.061 & 0.076 \\
\hline 2012_06_15 & 0.480 & 0.431 & 0.421 & 0.405 & 0.371 & 0.367 & 0.034 & 0.038 \\
\hline 20120629 & 0.731 & 0.671 & 0.660 & 0.681 & 0.620 & 0.599 & 0.061 & 0.082 \\
\hline $2012 \_07^{-} 27$ & 0.694 & 0.661 & 0.656 & 0.656 & 0.586 & 0.586 & 0.070 & 0.070 \\
\hline 2012_08_01 & 0.715 & 0.712 & 0.709 & 0.679 & 0.661 & 0.642 & 0.018 & 0.037 \\
\hline 2012_08_02 & 0.765 & 0.680 & 0.674 & 0.700 & 0.645 & 0.640 & 0.055 & 0.060 \\
\hline 2012_08_18 & 0.735 & 0.655 & 0.661 & 0.671 & 0.601 & 0.600 & 0.070 & 0.071 \\
\hline 2012_08_21 & 0.688 & 0.652 & 0.626 & 0.655 & 0.608 & 0.559 & 0.047 & 0.096 \\
\hline 2012_08_22 & 0.682 & 0.664 & 0.646 & 0.632 & 0.617 & 0.590 & 0.015 & 0.042 \\
\hline 2012_09_03 & 0.698 & 0.641 & 0.634 & 0.627 & 0.586 & 0.539 & 0.041 & 0.088 \\
\hline 2012_09_08 & 0.654 & 0.593 & 0.584 & 0.603 & 0.533 & 0.516 & 0.070 & 0.087 \\
\hline $2012^{-} 09^{-} 13$ & 0.622 & 0.589 & 0.537 & 0.507 & 0.503 & 0.443 & 0.004 & 0.064 \\
\hline $2012^{-} 09^{-} 17$ & 0.543 & 0.510 & 0.483 & 0.481 & 0.440 & 0.410 & 0.041 & 0.071 \\
\hline $2012^{-} 09-21$ & 0.499 & 0.438 & 0.464 & 0.426 & 0.384 & 0.360 & 0.042 & 0.066 \\
\hline 2012_10_10 & 0.368 & 0.350 & 0.314 & 0.310 & 0.298 & 0.241 & 0.012 & 0.069 \\
\hline 2012_11_26 & 0.211 & 0.198 & 0.123 & 0.149 & 0.145 & 0.118 & 0.004 & 0.031 \\
\hline $2012^{-} 12^{-} 07$ & 0.173 & 0.158 & 0.104 & 0.133 & 0.106 & 0.093 & 0.027 & 0.040 \\
\hline
\end{tabular}

\section{RESULTS AND DISCUSSION}

Vegetation index of FY-3A/VIRR and FY3A/MERSI have a highly significant correlations with Terra/MODIS. But a certain differences also exist. Reasons for such differences are as below.

\subsection{The spectral response function of sensors is different.}

The spectral response functions of corresponding bands of FY-3A/VIRR and Terra/MODIS, FY$3 \mathrm{~A} / \mathrm{MERSI}$ and Terra/MODIS are respectively shown in figure 2 and figure 3 . Red band scope of Terra/MODIS is $620-670 \mu \mathrm{m}$, near-infrared band scope of Terra/MODIS is $841-867 \mu \mathrm{m}$. Red band scope of FY-3A/VIRR is $580-680 \mu \mathrm{m}$, near-infrared band scope of FY-3A/VIRR is $840-870 \mu \mathrm{m}$. Red band scope of FY-3A/MERSI is $600-700 \mu \mathrm{m}$, nearinfrared band scope of FY-3A/MERSI is 845$915 \mu \mathrm{m}$. To sum up, the spectral response function of corresponding band of Terra/MODIS is narrower than those of FY-3A/VIRR and FY-3A/MERSI. The spectral response function reflects sensors' response capability of light. The sensitivity of Terra/MODIS red band and near-infrared band towards spectral changes is stronger than that of FY-3A/VIRR and FY-3A/MERSI. 


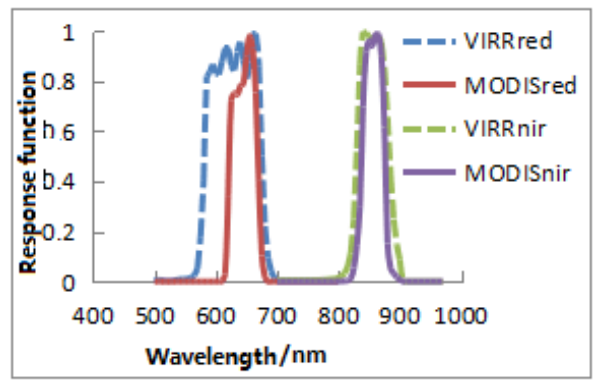

Figure 2. Comparison of spectral response function between FY-3A/VIRR and Terra/MODIS

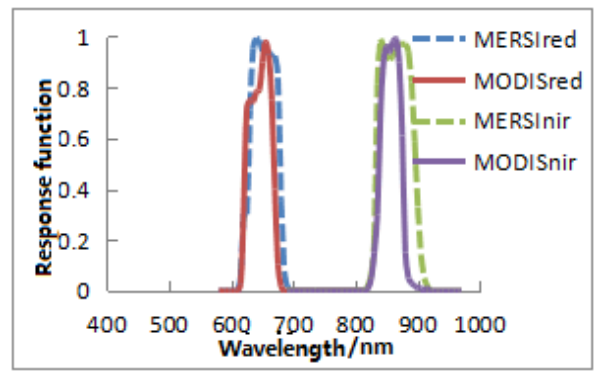

Figure 3. Comparison of spectral response function between FY-3A/MERSI and Terra/MODIS

\subsection{Radiometric calibration}

The calibration precision of FY-3A/VIRR and FY3A/MERSI is both lower than that of Terra/MODIS. The calibration parameters of each band of FY3A/VIRR and FY-3A/MERSI will have varying degrees of degeneration along with the changing of launch time. Terra/MODIS adopts on-orbit calibration. Its three on-orbit calibrations, which are sun scatter plate, sun scattering stability detector and spectral radiation calibration device, are used to calibrate the sun's reflection band. These calibrators can provide high-precision calibration results. Due to the uncertainty brought by calibration, NDVI from the data of Terra/MODIS can better reflect the growing situation of plants compared with that of FY3A/VIRR and FY-3A/MERSI.

\section{CONCLUSION}

This paper conducts a comparison of vegetation index from the data of FY-3A/VIRR, FY-3A/MERSI and Terra/MODIS. The reasons for the differences of NDVI were analyzed. The conclusions are as follows: First, vegetation indexes of FY-3A/VIRR, FY$3 \mathrm{~A} / \mathrm{MERSI}$ is very close to that of Terra/MODIS. All the correlation coefficients exceeded 0.99. But there are some differences of NDVI between FY3A/VIRR, FY-3A/MERSI and Terra/MODIS. NDVI of Terra/MODIS is higher than that of FY-3A/VIRR and FY-3A/MERSI. Vegetation index of Ter- $\mathrm{ra} / \mathrm{MODIS}$ is more sensitive, and it can get more rich information of vegetation. Second, the differences in spectral response function and calibration precision of FY-3A/VIRR, FY-3A/MERSI and Terra/MODIS has certain influences on vegetation index. In practical application, cross-calibration can be adopted to narrow the differences of vegetation index from the data of FY-3A/VIRR and Terra/MODIS as well as FY-3A/MERSI and Terra/MODIS.

\section{ACKNOWLEDGEMENTS}

This work was supported by the National High Technology Research and Development Program of China under Grant 2013AA12A301.

\section{REFERENCES}

Gallok, Lei J, I Reed B, etal. 2005. Multi-platform comparisons of MODIS and AVHRR nomalized difference vegetation data, Remote Sensing of Environment, 99: 221-231.

Jun, Y. Chaohua, D. Neimeng, L. 2009. FY-3A the new generation polar-oribiting meteorological satellite of China, Acta Meteorologica, 67: 501-509.

Kaufman Y J, Tanre D. 1992. Atmospherically resistant vegetation index (ARVI) for EOS-MODIS, IEEE Trans. on Geoscience and Remote Sensing, 30: 261-270.

Tomoaki M. 2000. Evaluation of sensor calibration uncertainties on vegetation indices for MODIS, IEEE Trans.on Geoscience and Remote Sensing, 38: 1399-1409.

Xueyi, Z. 2009. Introduction of several vegetation indices based on EOS/MODIS, Journal of Anhui Agri Sci, 37, $12842-12845,12850$. 